\title{
CONDICIONANTES DE LAS GRIETAS INTERNAS DENTRO DE LOS ANILLOS DURANTE EL SECADO DE PINO RADIATA
}

\section{SOME FACTORS AFFECTING THE WHITING-RING INTERNAL CHECKING DURING DRYING OF RADIATA PINE}

\author{
José Vera Sepúlveda ${ }^{1}$, Rubén A. Ananías², Juan E. Díaz-Vaz³.
}

\section{RESUMEN}

Se estudian las grietas internas dentro de los anillos de madera inicial durante el secado industrial del pino radiata. Se determina el porcentaje de las grietas internas y se analizan algunos factores condicionantes de las grietas internas durante el secado industrial a temperaturas convencionales.

Los ensayos de secado a temperaturas convencionales son realizados en un horno convectivo de $150 \mathrm{~m}^{3}$ de capacidad, operando en una planta industrial de la Octava Región (Chile). El diseño experimental incluye como factores variables, el sitio de procedencia de la madera (arcilla, arena), el tipo de madera (central, lateral y transición) y el tipo de corte (radial, tangencial y mixto).

Los resultados indican que el porcentaje de grietas internas varía entre 1 y $18 \%$ y que el sitio de procedencia de la madera y el tipo de madera condicionan significativamente el porcentaje de grietas internas durante el secado industrial a temperaturas convencionales del pino radiata.

Palabras clave: Secado convencional, grietas internas, Pinus radiata.

\begin{abstract}
The internal checking during the industrial drying of radiata pine is studied. The percentage of internal checking is determined and some factors influencing the internal checking are analyzed during the industrial drying at conventional temperatures.

The runs are made in a $150 \mathrm{~m}^{3}$ capacity dry kiln in an industrial plant of the Eighth Region (Chile). The experimental design includes as variables, the origin site of the wood (clay, sand), the wood localization (central-wood, lateral-wood and transition-wood) and the type of cut (radial, tangential and mixed).

The results indicate that the percentage of internal checking varies between 1 and $18 \%$ and that the origin site of the wood and the wood localization significantly condition the percentage of internal checking during the radiata pine industrial drying at conventional temperatures.
\end{abstract}

Keywords: wood drying, internal checking, Pinus radiata

\footnotetext{
${ }^{1}$ Centro de Formación Técnica Lota-Arauco. e-mail: jvera@ cftlotarauco.cl

2Depto. Ingeniería en Maderas. Fac. Ingeniería. Universidad del Bío-Bío. e-mail: ananias@ubiobio.cl

${ }^{3}$ ITPF. Fac. Ciencias Forestales. Universidad Austral. e-mail: jdiazvaz@uaustral.cl

Autor para correspondencia: ananias@ubiobio.cl

Recibido: 08 de marzo 2007. Aceptado: 14 de junio de 2007.
} 


\section{INTRODUCCION}

En el presente estudio se aborda la frecuencia de aparición del defecto de grieta interna dentro de los anillos de madera inicial. La grieta interna dentro de los anillos se origina como resultado de la tensión capilar que supera la resistencia mecánica de la madera, especialmente en células de madera temprana, donde la pared celular es más delgada. Evidencias de que la falla ocurre en la lamela media compuesta de las traqueidas de madera inicial ha sido reportada (Putoczki et al. 2006). Tal como se muestra en la Figura 1, estas grietas internas dentro de los anillos se forman dentro del anillo de crecimiento de madera inicial, tienen una forma lenticular y se encuentran de a una o varias juntas en una sección (Booker and Haslett, 2001; Pang et al. 1999; Putoczky et al. 2006)). Igualmente se da una interrelación entre la aparición de grietas internas en la madera y el colapso; ya que ambos ocurren como resultado de la tensión del líquido desarrollada por el movimiento del agua al salir de los lúmenes de las células (Miller and Simpson, 1992; Booker, 1994; Booker and Koga, 2003). No obstante Potaczki et al. 2006, muestra evidencias de células con grietas internas sin colapso y viceversa.

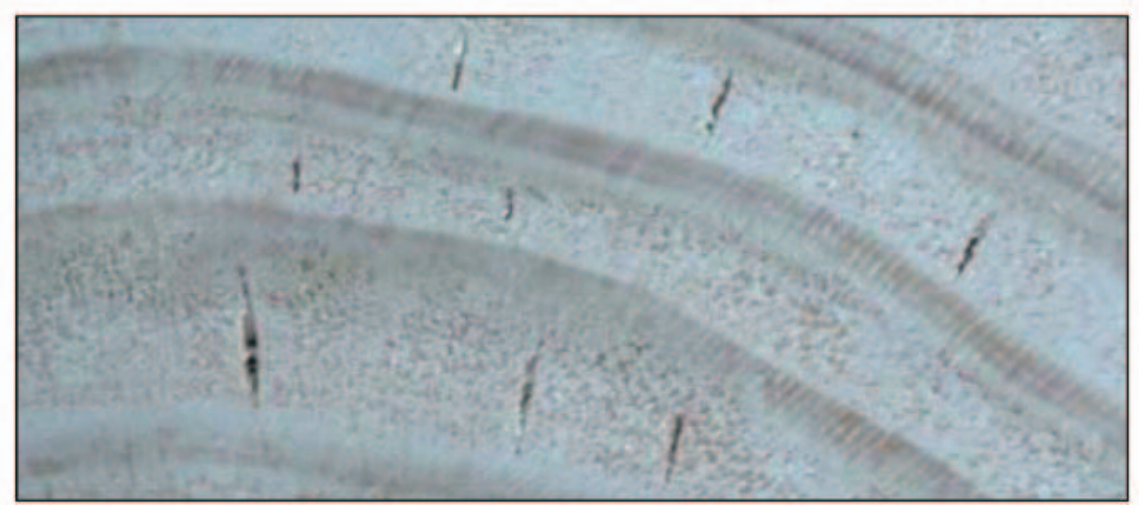

Figura 1: Grietas internas dentro de los anillos en pino radiata

Algunos factores condicionantes de las grietas internas dentro de los anillos son, la clase de sitio, los factores genéticos, la localización de la madera en el trozo y la intensidad del secado, entre otros (Cown and McConchie, 2000; Simpson et al. 2002; Ball et al. 2005a, b) .

Se ha evidenciado que sitios con deficiencia de nutrientes, particularmente de boro, presentan mayor propensión a las grietas internas dentro de los anillos, la deficiencia de boro siendo uno de los micronutrientes importantes participando en la generación de los primeros estados de la pared celular limitaría la elasticidad de la pared celular, favoreciendo la propensión a las grietas internas dentro de los anillos (Olykan, 2004; McLaren, 2002).

Además las grietas internas dentro de los anillos podrían tener una componente genética, cierto grado de heredabilidad de éstas ha sido reportado en la literatura (Kumar, 2004; FRI, 2003; Ball et al. 2001).

Por otra parte, las grietas internas dentro de los anillos son más frecuentes en el margen de los anillos duraminizados (McLaren, 2002; Cown and McConchie, 2000; Millar and Simpson, 1992), así como no se presentan en la madera de compresión (Ball et al. 2005a,b). Las grietas internas dentro de los anillos son más frecuentes en madera central entre los anillos 5 a 15 desde la médula (Miller and Simpson, 1992; McConchie 1999). 
El defecto aparece en forma esporádica, se intensifica con el secado y puede ser invisible en la superficie de la madera (Maclaren, 2002, Cown and McConchie, 2000), en ocasiones puede ser menor que el $1 \%$ del volumen de la madera, pero algunas veces puede afectar entre el 5 y $10 \%$ (Cown and McConchie, 2000; Simpson et al. 2002). Para reducir este tipo de grieta interna se sugiere bajar la temperatura de secado, el secado convencional puede reducir hasta en un cuarto las grietas internas dentro de los anillos en comparación al secado por alta temperatura (Haslett, 1998); Simpson et al. 2002). No obstante la calidad de la materia prima utilizada condiciona la presencia de grietas internas dentro de los anillos (Simpson et al. 2002)

Usualmente las grietas internas dentro de los anillos no se observan sino hasta después que la madera ha sido manufacturada, durante el maquinado o ensamble de productos terminados (Miller and Simpson, 1992; Haslett, 1998).

Debido a este defecto, se genera un aumento en los costos de fabricación y un aumento en la fracción defectuosa de los productos finales. Esto se refleja claramente en el proceso de remanufactura y en madera destinada a exportación (molduras), uno de los mercados más importantes para la industria. La presencia de grietas internas dentro de los anillos en la madera temprana es uno de los mayores defectos que degrada la calidad del Pinus radiata, lo que se estima ocasiona pérdidas millonarias (Putoczki et al. 2006).

Por otra parte, se pueden identificar árboles con tendencia a la aparición de esta grieta interna dentro de los anillos y hay un porcentaje de ellos, que tienen grietas internas entre los anillos antes de ser aserrados (Simpson et al. 2002; Ball et al. 2005a,b Vera, 2005).

Este trabajo tiene como objetivos estudiar la influencia de la procedencia del árbol, el tipo de suelo, el tipo de corte en la madera y el tipo de madera, sobre las grietas internas dentro de los anillos durante el secado industrial del pino radiata de $46 \mathrm{~mm}$ de espesor.

\section{METODOLOGIA}

De acuerdo a los antecedentes preliminares recogidos en la industria, se identifican inicialmente como factores que condicionan las grietas internas dentro de los anillos: Las zonas de procedencia de la madera (2), el tipo de corte de la madera (3) y el tipo de madera o la localización de la madera en el trozo (3), con lo cual se ejecutan 18 tratamientos, distribuidos aleatoriamente en 6 cargas de secado (experimento 1). La mezcla de factores se muestra en la tabla 1.

Identificación de las piezas ensayadas. Se tomó una muestra de un $15 \%$ de cada carga en cada uno de los experimentos. Se procedió a enumerar las piezas en ambos extremos de estas de acuerdo a la mezcla de factores a la cual correspondería para tener su trazabilidad en los procesos de secado, cepillado y trozado. 
Tabla 1. Mezcla de factores experimento 1

\begin{tabular}{|c|c|c|c|}
\hline Tratamiento & Procedencias & Tipo de corte & Tipo de madera \\
\hline 1 & Zona 1 & Tangencial & Central \\
\hline 2 & Zona 1 & Tangencial & Lateral \\
\hline 3 & Zona 1 & Tangencial & Transición \\
\hline 4 & Zona 1 & Radial & Central \\
\hline 5 & Zona 1 & Radial & Lateral \\
\hline 6 & Zona 1 & Radial & Transición \\
\hline 7 & Zona 1 & Mixto & Central \\
\hline 8 & Zona 1 & Mixto & Lateral \\
\hline 9 & Zona 1 & Mixto & Transición \\
\hline 10 & Zona 2 & Tangencial & Central \\
\hline 11 & Zona 2 & Tangencial & Lateral \\
\hline 12 & Zona 2 & Tangencial & Transición \\
\hline 13 & Zona 2 & Radial & Central \\
\hline 14 & Zona 2 & Radial & Lateral \\
\hline 15 & Zona 2 & Radial & Transición \\
\hline 16 & Zona 2 & Mixto & Central \\
\hline 17 & Zona 2 & Mixto & Lateral \\
\hline 18 & Zona 2 & Mixto & Transición \\
\hline
\end{tabular}

De acuerdo al estudio mencionado más arriba (experimento 1), se estableció que la Zona 1 tenía el mayor porcentaje de grietas internas dentro de los anillos, por lo que se procedió a una caracterización de esta madera. Para esta parte del estudio (experimento 2), se consideraron como factores variables, el tipo de suelo (2), la altura en el árbol (2) y el tiempo de estadía de la madera aserrada en el patio (2), por lo cual se ejecutaron un total de 8 tratamientos, distribuidos aleatoriamente en 4 cargas de secado (Tabla 2).

Tabla 2. Mezcla de factores ensayos experimento 2

\begin{tabular}{|c|c|c|c|}
\hline Tratamiento & Tipo de suelo & Altura en el árbol & Tiempo en patio \\
\hline 1 & Arena & Copa & $>10$ días \\
\hline 2 & Arena & Copa & $<10$ días \\
\hline 3 & Arena & Base & $>10$ días \\
\hline 4 & Arena & Base & $<10$ días \\
\hline 5 & Greda & Copa & $>10$ días \\
\hline 6 & Greda & Copa & $<10$ días \\
\hline 7 & Greda & Base & $>10$ días \\
\hline 8 & Greda & Base & $<10$ días \\
\hline
\end{tabular}


Secado. Las piezas preparadas y marcadas fueron enseguida secadas en un horno industrial de $150 \mathrm{~m}^{3}$ de capacidad (Figura 2), bajo un programa usualmente utilizado en la empresa donde se realizaron las experiencias (Los Angeles, VIII Región, Chile).

Determinación del porcentaje de grietas internas dentro de los anillos en proceso de trozado. Luego del secado la madera fue cepillada en las 4 caras y transportada al área de trozado. Mediante despuntado de $5 \mathrm{~cm}$ de cada extremo se contaron y midieron las grietas internas dentro de los anillos de cada lado despuntado. Se evaluó la profundidad y el número de grietas, luego si el despunte presentaba grietas se continuaba con el despunte de otros $5 \mathrm{~cm}$, sino se considera libre de grietas. Con estos datos se obtuvó el porcentaje en volumen de grietas internas dentro de los anillos para la madera en estudio.

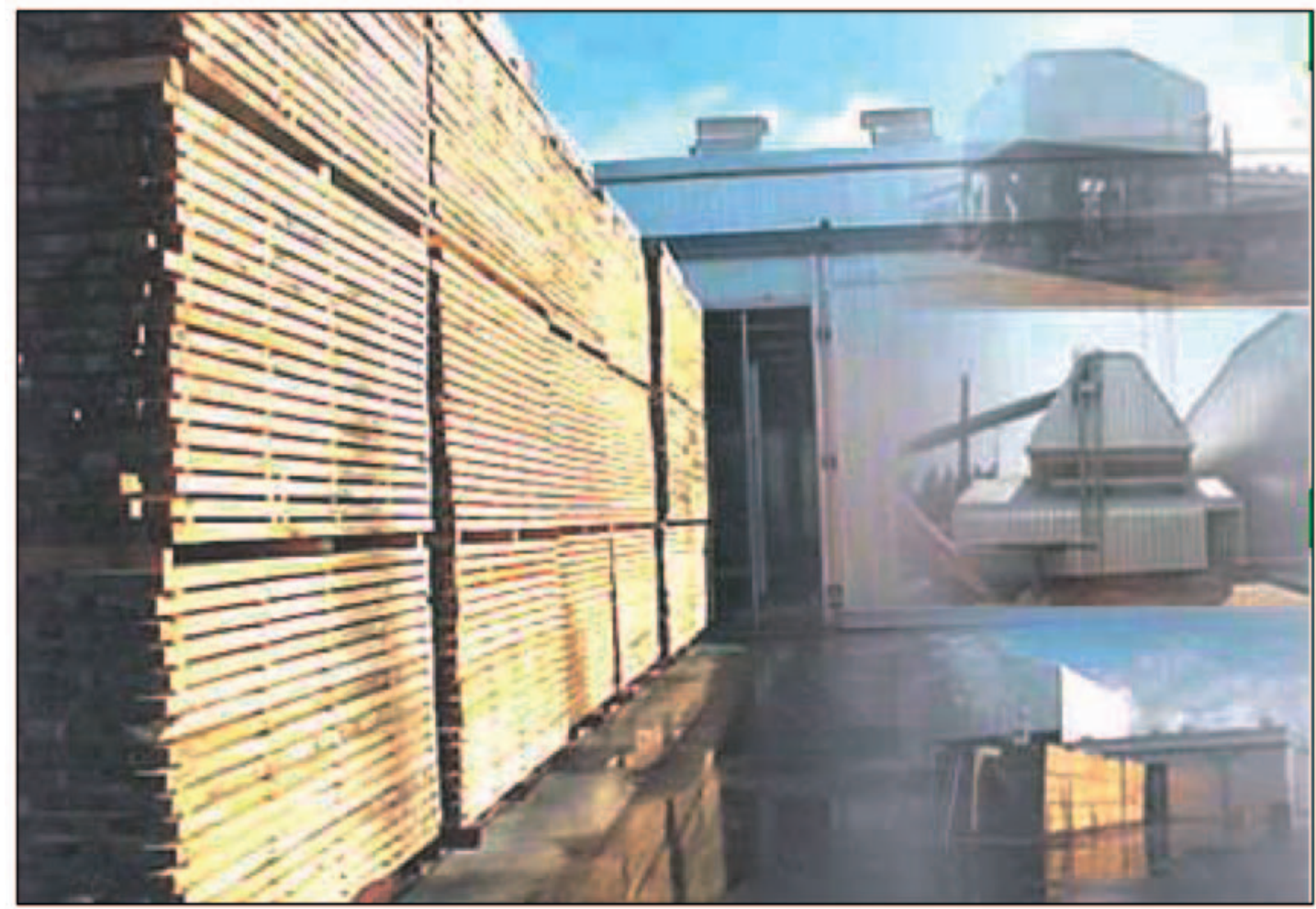

Figura 2: Horno de secado industrial

Tratamiento estadístico. Los resultados fueron analizados mediante un ANDEVA modelo factorial con aleatorización completa, utilizando un software comercial. Las pruebas de Barlett y de KolmogoroffSmirnow fueron realizadas para asegurar la homogeneidad de la varianza y la normalidad de los datos respectivamente.

Considerando que la variable respuesta Y corresponde al porcentaje de piezas con grietas internas dentro de los anillos, el modelo estadístico se expresa como:

$$
\mathrm{Y}_{\mathrm{ijkl}}=\mu+\alpha_{\mathrm{i}}+\beta_{\mathrm{j}}+\delta_{\mathrm{k}}+(\boldsymbol{\beta})_{\mathrm{ij}}+(\boldsymbol{\alpha})_{\mathrm{ik}}+(\boldsymbol{\beta})_{\mathrm{jul}}+\left(\boldsymbol{\beta} \quad{ }_{\mathrm{ijk}}+\varepsilon_{\mathrm{ijkl}(1)}\right.
$$

Siendo $\mu$ el promedio general, $\boldsymbol{\varepsilon}$ el error de las observaciones y los factores $\alpha, \beta$ y $\gamma$ los indicados en la tabla 3. 
Tabla 3. Significado de los factores considerados en la ecuación 1

\begin{tabular}{|c|c|c|}
\hline Factor & Estudio 1 & Estudio 2 \\
\hline$\alpha_{\mathrm{i}}$ & Procedencia & Tipo de suelo \\
\hline $\mathrm{i}$ & 1,2 & 1,2 \\
\hline$\beta_{\mathrm{j}}$ & Tipo de corte & Altura en el árbol \\
\hline $\mathrm{j}$ & $1,2,3$ & 1,2 \\
\hline$\gamma_{\mathrm{k}}$ & Tipo de madera & Tiempo en patio \\
\hline $\mathrm{k}$ & $1,2,3$ & 1,2 \\
\hline
\end{tabular}

\section{RESULTADOS}

En la tabla 4 se muestran los porcentajes de grietas internas dentro de los anillos obtenidos de los 18 experimentos realizados. Se observa que el volumen de grietas internas dentro de los anillos varía entre $0,8 \%$ y $18 \%$.

Tabla 4. Grietas internas dentro de los anillos (\%) durante ensayos secado industrial de pino radiata

\begin{tabular}{|c|c|c|c|}
\hline Procedencias & $\begin{array}{c}\text { Tipo de } \\
\text { corte }\end{array}$ & $\begin{array}{c}\text { Tipo de } \\
\text { madera }\end{array}$ & $\begin{array}{c}\text { Grietas internas dentro de los anillos } \\
\mathbf{( \% )}\end{array}$ \\
\hline Zona 1 & Tangencial & Central & 8,9 \\
Zona 1 & Tangencial & Lateral & 9,8 \\
Zona 1 & Tangencial & Transición & 7,3 \\
Zona 1 & Radial & Central & 15,5 \\
Zona 1 & Radial & Lateral & 13,6 \\
Zona 1 & Radial & Transición & 14,8 \\
Zona 1 & Mixto & Central & 8,7 \\
Zona 1 & Mixto & Lateral & 18,0 \\
Zona 1 & Mixto & Transición & 7,0 \\
Zona 2 & Tangencial & Central & 8,1 \\
Zona 2 & Tangencial & Lateral & 12,2 \\
Zona 2 & Tangencial & Transición & 12,5 \\
Zona 2 & Radial & Central & 6,6 \\
Zona 2 & Radial & Lateral & 2,9 \\
Zona 2 & Radial & Transición & 0,8 \\
Zona 2 & Mixto & Central & 11,1 \\
Zona 2 & Mixto & Lateral & 6,2 \\
Zona 2 & Mixto & Transición & 8,4 \\
\hline
\end{tabular}


De acuerdo a la tabla 5, se evidencia la importancia de cada uno de los factores considerados en el diseño del experimento, así como también la importancia de cada una de las interacciones entre estos factores.

Tabla 5. Contribución de los factores a la formación de las grietas internas dentro de los anillos durante el secado de pino radiata.

\begin{tabular}{|c|c|}
\hline FACTOR & \% DE CONTRIBUCION \\
\hline Procedencia (A) & 21,9 \\
Corte (B) & 1,3 \\
Madera (C) & 46,2 \\
AB & 15,3 \\
AC & 1,4 \\
BC & 1,9 \\
\hline
\end{tabular}

Se aprecia que los factores con mayor participación en el presente experimento son: la procedencia de la madera $(21.9 \%)$ y el tipo de madera $(46.2 \%)$. Estos factores son estadísticamente significativos con un nivel de confianza de $95 \%(\alpha=0.05)$. Las interacciones entre procedencia y el tipo de madera también evidencian la mayor participación $(15.3 \%)$. Las interacciones de tercer orden no son significativas.

En la figura 3, se observa que el tipo de corte que presenta un mayor efecto sobre la cantidad de grietas internas dentro de los anillos es el corte radial, seguido del mixto y el tangencial. Los resultados entregados se ajustan a la literatura, ya que los radios leñosos y la lamela media compuesta pueden tener influencia en la aparición de grietas internas dentro de los anillo, debido a que estas zonas presentan una reorganización particular de las paredes celulares sin microfibrillas limitando su flexibilidad y resistencia mecánica (Miller \& Simpson 1992; Simpson et al. 2002; Putoczki et al. 2006). La tensión capilar actuando sobre las paredes celulares es probablemente más intensa en el plano radial debido al mayor flujo capilar tangencial, como consecuencia a la mayor cantidad y tamaño de las punteaduras sobre las paredes radiales de las fibras, lo que favorece la aparición de grietas internas entre los anillos en la dirección de los radios leñosos. La hipótesis anterior es reforzada por la menor flexibilidad y resistencia mecánica de las paredes tangenciales en comparación a las radiales (Booker and Koga, 2003; Putoczki et al. 2006). 


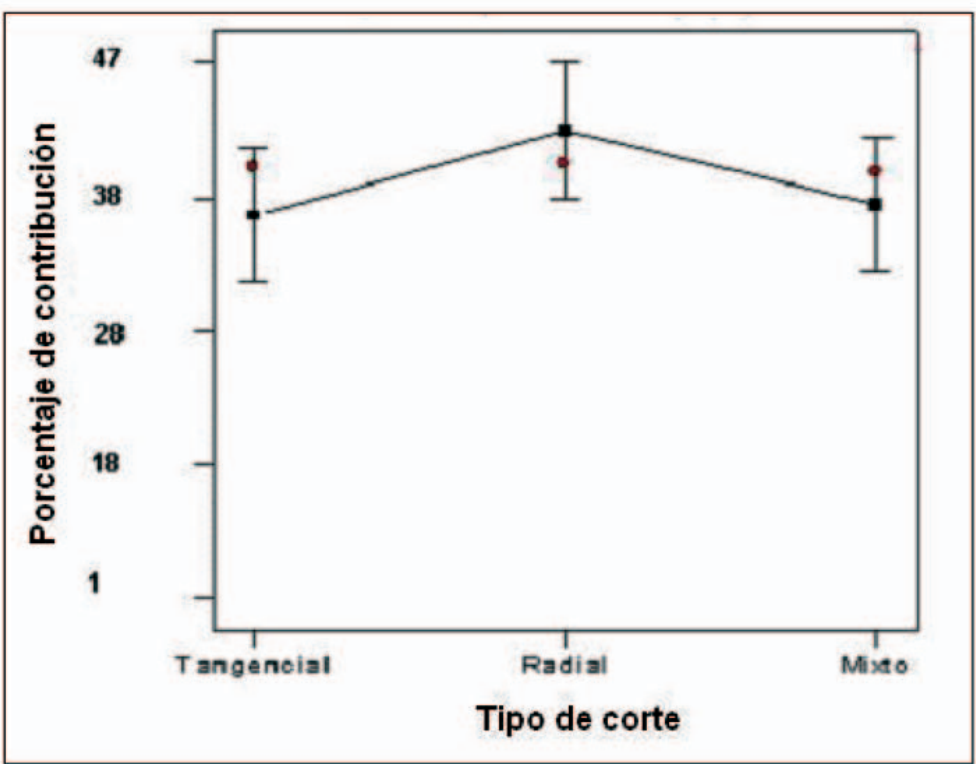

Figura 3: Efecto del tipo de corte sobre las grietas internas dentro de los anillos (\$Valores reales \$ Valores teóricos)

En la figura 4 se aprecia el efecto del tipo de madera sobre las grietas internas dentro de los anillos. El tipo de madera es un factor relevante a la hora de mejorar el secado industrial de pino radiata de 46 $\mathrm{mm}$ de espesor, de acuerdo a lo mostrado en la figura $3 \mathrm{y}$ al porcentaje de contribución obtenido $(46.2 \%)$.

En el área de secado de la empresa donde se realiza este trabajo, los paquetes de madera que se preparan para el secado, contienen madera central y lateral, no contemplándose ninguna separación entre estos dos tipos de madera. De acuerdo con este estudio, es aconsejable separar la madera central de la lateral para disminuir la aparición de grieta interna dentro de los anillos y mejorar el secado industrial.

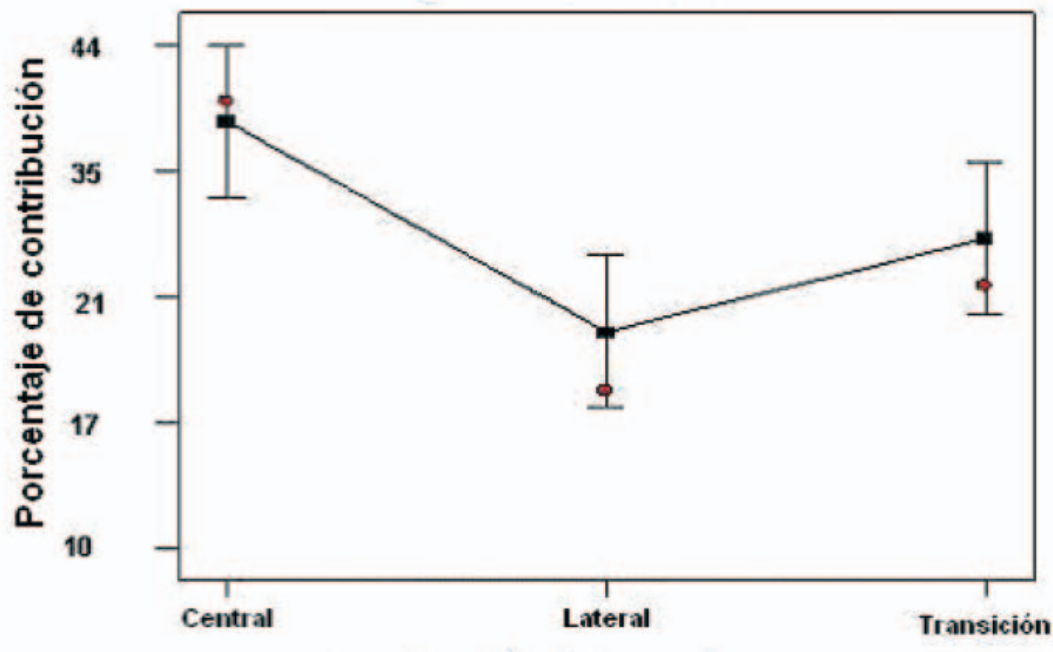

Tipo de madera

Figura 4: Efecto del tipo de madera sobre las grietas internas dentro de los anillos ( $\$$ Valores reales \$Valores teóricos) 
La madera central y lateral posee características diferentes (madera juvenil/madera adulta), lo que las afecta durante el secado bajo un mismo programa. Además, si se separa la madera central de la madera lateral, se ahorra en tiempo; ya que para la madera lateral se puede utilizar un programa que demande una menor cantidad de tiempo real de secado.

En la figura 5 se observa, el efecto de la interacción de los factores procedencia-tipo de madera sobre las grietas internas dentro de los anillos. El comportamiento es similar al análisis de los factores por separado, debido a que el mayor porcentaje de grietas internas dentro de los anillos lo presenta la Zona 1 junto con la madera central, y el menor porcentaje de grietas internas lo presenta la Zona 2 con madera lateral.

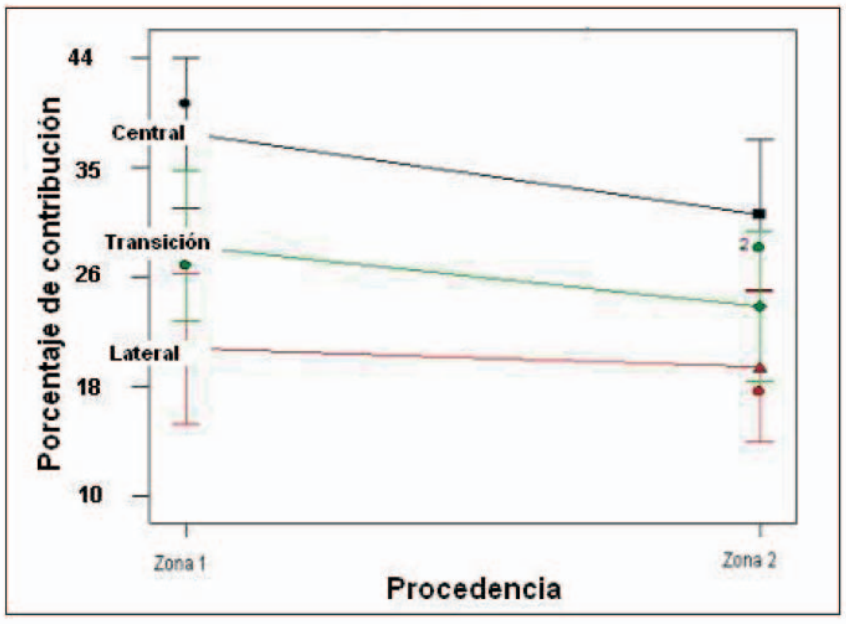

Figura 5: Efecto de la interacción procedencia-tipo de madera sobre las grietas internas dentro de los anillos. ( Valores reales \$ Valores teóricos)

La interacción de ambos factores, procedencia y tipo de madera, no varían demasiado con respecto al análisis de los factores por separado, como se observa en la figura 6, el mayor efecto sobre las grietas internas dentro de los anillos es producido en la Zona 1 en combinación con el corte radial.

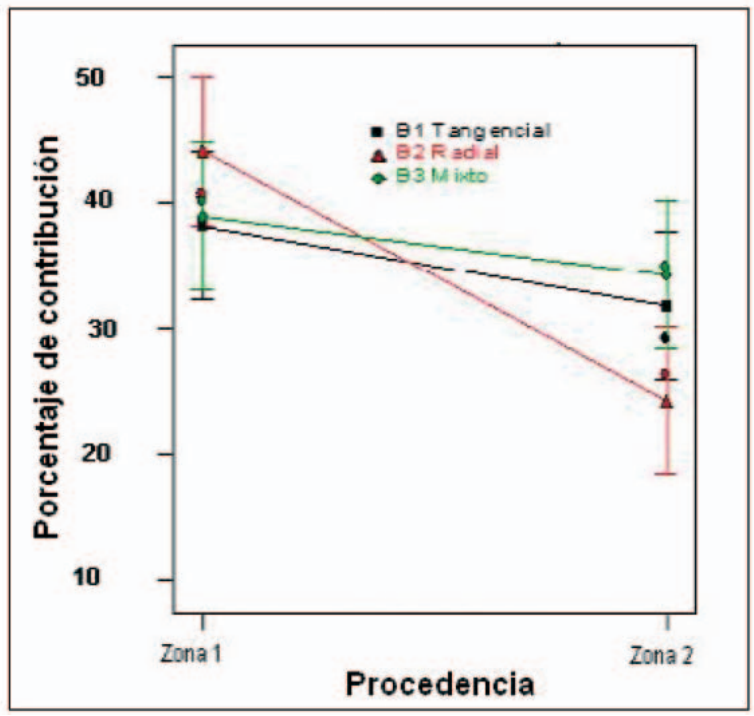

Figura 6: Efecto de la interacción procedencia-tipo de corte sobre las grietas internas dentro de los anillos ( $\$$ Valores reales $\$$ Valores teóricos). 
Como la procedencia de la madera resulta tener un efecto significativo sobre las grietas internas dentro de los anillos y es la Zona 1 la que presenta el mayor volumen de este defecto, es que se analiza a continuación la influencia de dos tipos de suelo de la Zona 1 (arena y arcilla) con la altura en el árbol y el tiempo en patio. En la tabla 6 se muestran los resultados del porcentaje de grietas internas dentro de los anillos obtenidos para la Zona 1.

Tabla 6. Grietas internas dentro de los anillos en ensayos de secado industrial de pino radiata proveniente de la Zona 1.

\begin{tabular}{|c|c|l|c|}
\hline Tipo de suelo & $\begin{array}{c}\text { Altura en el } \\
\text { árbol }\end{array}$ & \multicolumn{1}{|c|}{$\begin{array}{c}\text { Tiempo en } \\
\text { patio }\end{array}$} & $\begin{array}{c}\text { Grietas internas entre los anillos } \\
\mathbf{( \% )}\end{array}$ \\
\hline Arena & Copa & $\begin{array}{l}\text { Más de 10 días } \\
\text { Menos de 10 }\end{array}$ & 19,8 \\
Arena & Copa & días & 20,3 \\
Arena & Base & Más de 10 días & 13,7 \\
Arena & Base & Menos de 10 & 10,6 \\
Greda & Copa & Más de 10 días & 14,5 \\
Greda & Copa & Menos de 10 & 12,6 \\
Greda & Base & Más de 10 días & 7,7 \\
Greda & Base & Menos de 10 & 5,1 \\
\hline
\end{tabular}

En este caso se observa que la proporción de madera con grietas internas dentro de los anillos varió entre $5,1 \%$ y $19,8 \%$. La importancia de los factores participantes en el experimento se observa en la tabla 7.

Tabla 7. Contribución de los factores de la zona 1

\begin{tabular}{|c|c|}
\hline FACTOR & \% DE CONTRIBUCION \\
\hline Suelo (D) & 37,4 \\
Altura (E) & 56,4 \\
Tiempo (F) & 3,9 \\
DE & 0,2 \\
DF & 0,4 \\
EF & 1,6 \\
\hline
\end{tabular}

Como se observa, los factores D y E, presentan una importante contribución al experimento (37.4 $\%$ y 56.4\% respectivamente), no así el factor E, el cual presentó un porcentaje de contribución del $3.9 \%$. En este caso, D y E resultaron ser estadísticamente significativos. Las interacciones de segundo $\mathrm{y}$ tercer orden no son significativas.

De acuerdo con la figura 7, la madera proveniente de suelo arenoso muestra una mayor contribución sobre las grietas internas dentro de los anillos en comparación a la madera creciendo en suelo gredoso. La contribución de este factor (37.4\%), muestra la importancia de la selección de trozos que presenten 
una baja tendencia a las grietas internas dentro de los anillos. La diferencia en los resultados de este factor, tiene probablemente su origen en la distribución y tipo de nutrientes existentes en los distintos tipos de suelo. Resultados preliminares muestran una mayor tendencia a las grietas en trozas de madera proveniente de suelos arenosos y de la copa de los árboles (Vera, 2005).

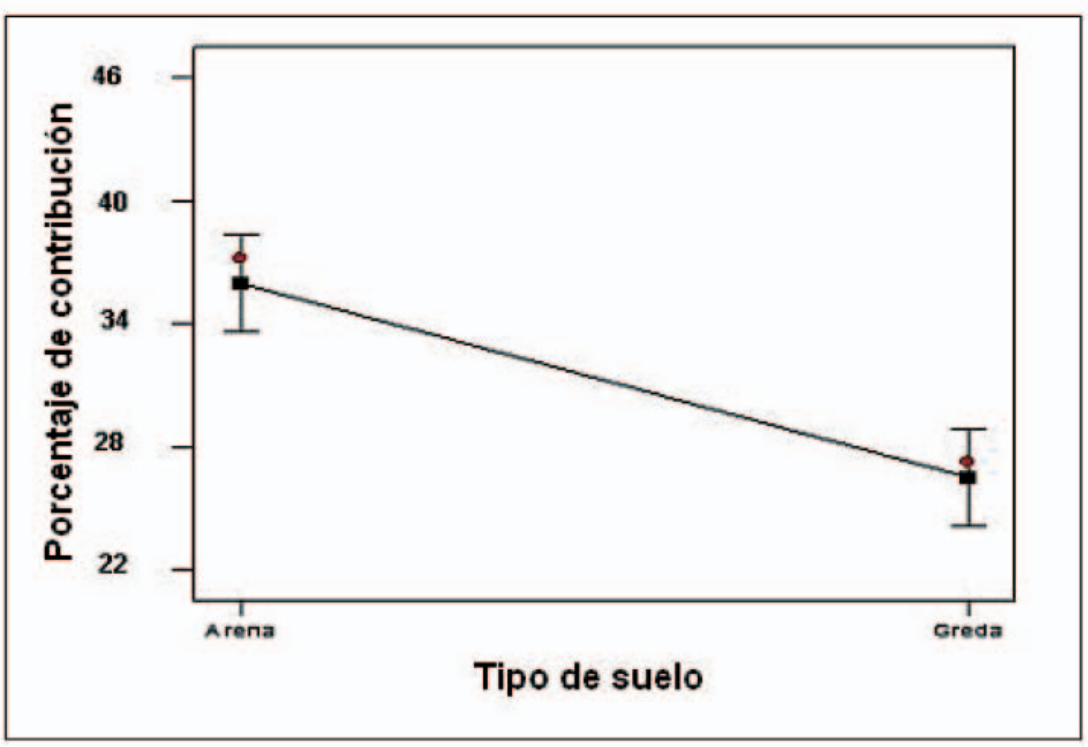

Figura 7: Efecto del tipo de suelo sobre las grietas internas dentro de los anillos ( Valores reales $\$$ Valores teóricos)

El área de secado en la industria generalmente separa las procedencias de la madera antes de ser ingresada a las cámaras de secado; pero al igual como lo hacen con la madera central y lateral, aplican exactamente el mismo programa de secado a ambas procedencias, incluso en los meses mas lluviosos en que la diferencia de grietas internas dentro de los anillos es más notoria entre ambas procedencias de la madera. De acuerdo a esto, una solución paralela a la "separación entre madera lateral y central" es además separar por procedencias, aplicar programas de secado distintos a ambas procedencias, optimizando tanto el tiempo de secado y la tendencia a la aparición de grietas internas dentro de los anillos.

En la figura 8 se observa que los trozos provenientes de la base del árbol presentan una menor contribución de grietas internas dentro de los anillos que los trozos extraídos desde la copa del árbol. 


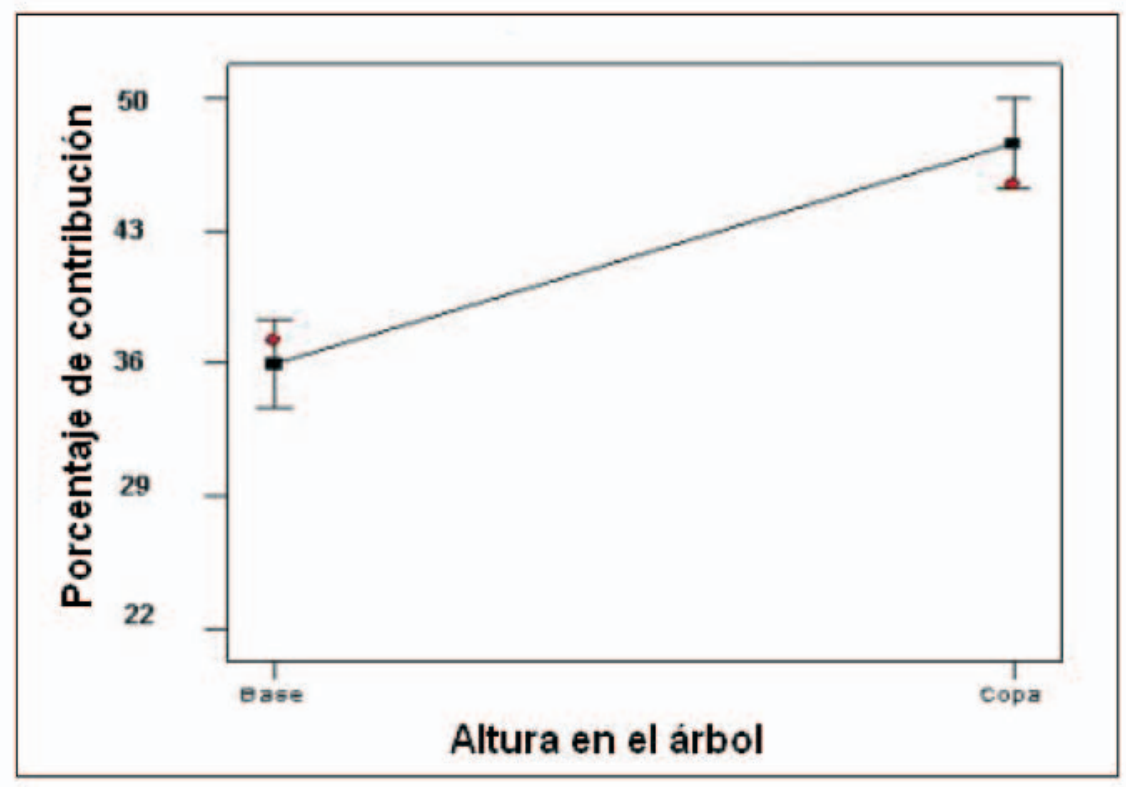

Figura 8: Efecto de la altura en el árbol sobre las grietas internas dentro de los anillos.

( Valores reales \$ Valores teóricos)

El presente factor presenta el porcentaje de contribución más alto (56.4\%). Esto indica que para madera con menor proporción de grietas internas dentro de los anillos, es recomendable utilizar madera proveniente de trozos de la base del árbol (primer corte).

Lo anterior se puede explicar, debido a que el árbol presenta un diámetro mayor en la base, lo que conlleva a que de esta parte se puede obtener mayor cantidad de madera lateral; que de acuerdo al experimento inicial, esta madera lateral presenta menor porcentaje de grietas que la madera central, como consecuencia probablemente de una menor presencia de madera juvenil hacia la base del árbol (madera juvenil/madera adulta).

Este factor también esta ligado con la variable tipo de madera, ya que al procesar trozos con un mayor diámetro, se obtiene mayor cantidad de madera lateral, limitando la aparición de grietas internas dentro de los anillos. Esta hipótesis, se ve favorecida además por la evidencia que las grietas internas dentro de los anillos se localizan principalmente en los márgenes de los anillos duraminizados (McLaren, 2002; Cown and McConchie, 2000; Miller and Simpson, 1992).

Por otra parte, a pesar de que el tiempo de estadía en patio no resulta ser significativo estadísticamente en el experimento, de acuerdo a la figura 9 se puede señalar que la madera con estadía en patio mayor a 10 días, favorece el porcentaje de grietas internas dentro de los anillos en comparación a la madera fresca. 


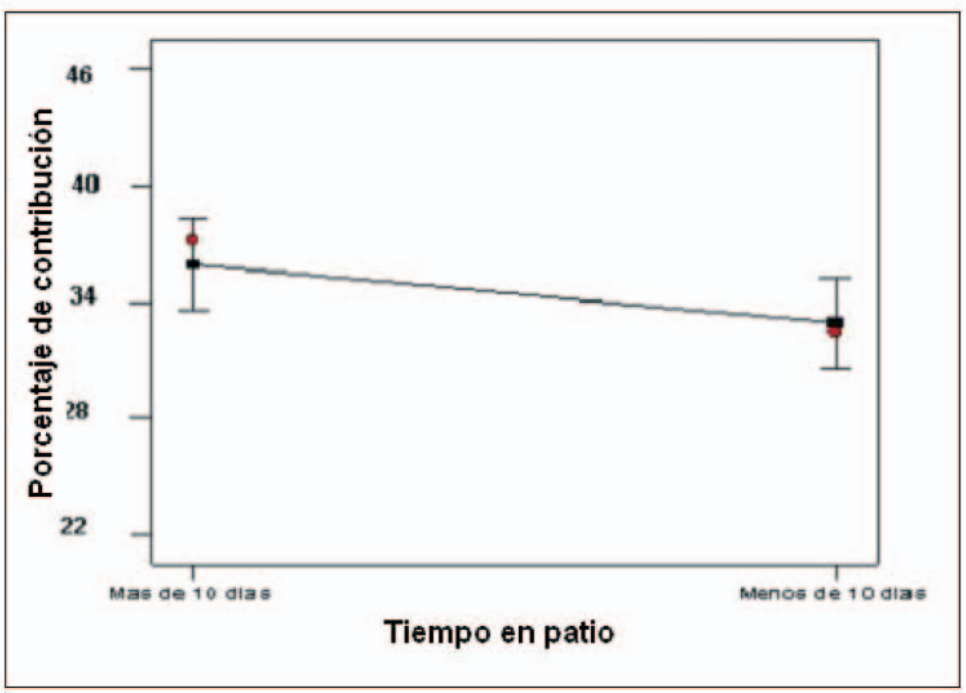

Figura 9: Efecto del tiempo de almacenamiento de la madera en patio sobre las grietas internas entre los anillos ( Valores reales \$ Valores teóricos)

Es probable que la madera con mayor tiempo en patio disminuya su contenido de humedad, en forma heterogénea, favoreciendo la aparición de las grietas internas dentro de los anillos durante el secado.

\section{CONCLUSIONES}

El porcentaje de grietas internas dentro de los anillos durante el secado industrial del pino radiata es significativamente condicionado por la procedencia, el tipo de madera y la altura en el árbol.

La madera proveniente de sitio arenoso presenta un porcentaje significativamente mayor de grietas internas dentro de los anillos que la madera proveniente de sitio gredoso.

El porcentaje de grietas internas dentro de los anillos es significativamente mayor en madera central. La madera extraída de la copa del árbol tiene un porcentaje significativamente mayor de grietas internas dentro de los anillos.

\section{AGRADECIMIENTOS}

Los autores agradecen el apoyo para realizar este trabajo y divulgar sus resultados a Don Oscar MATTHEI, Ingeniero Civil en Industrias Forestales y ex Gerente de Operaciones de la planta Remanufactura de Maderas Ltda. 


\section{BIBLIOGRAFIA}

Ball, R.D.; McConchie, M.; Cown, D.J. 2005a. Intra-ring checking in a study of 29 clonesevidence for associations with SilviScan variables. Fifth Workshop IUFRO S5.01.04, Waiheke Island, Auckland, New Zealand. 9pp.

Ball, R.D.; McConchie M.; Cown, D.J. 2005b. Evidence for associations between SilviScanmeasured wood properties and intraring checking in a study of twenty-nine 6-year-old Pinus radiata. Can. J. For. Res. 35:1156-1172.

Ball, R.D.; McConchie M.; Cown, D.J. 2001. Heritability of internal checking in pinus radiataevidence and preliminary estimates. New Zealand Journal of Forestry Science 31(1):78-87.

Booker, R.E, 1994. Internal checking and collapse which comes first? NZ Forest Research Institute. $4^{\text {th }}$ IUFRO International Wood Drying Conference, p. 133-140.

Booker, R.E.; Koga, S. 2003. How collapse and internal checking happen in boards during drying. Wood Process News 33: 3-5.

Booker, R.E.; Haslett, A.N. 2001. Acoustic emission study of within-ring internal checking in radiata pine. The E-Journal of Nondestructive Testing of Wood 6(3): 1-7.

Booker, R.; Haslett, A.N. 1994. Internal checking in radiate pine monitored by acoustic emission. $4^{\text {th }}$ IUFRO International Wood Drying Conference, p. 345.

Cown, D. J.; McConchie, D. 2000. Internal checking. Wood Processing Newsletter. Forest Research Issue $\mathrm{N}^{\circ} 27.2 \mathrm{pp}$.

Fri. 2003. What effects do genetics and site have on internal checking?. Forest Research Newsletter $\mathrm{N}^{\mathrm{o}} 4,1 \mathrm{pp}$.

Haslett, A.N. 1998. Drying random width lumber for remanufacturing. New Zealand Forest Research Institute. $10 \mathrm{pp}$.

Kininmorth, J.A. 1991. Properties and uses of New Zealand radiata pine. New Zealand Ministry of Forestry, Forest Research Institute.

Kumar, S. 2004. Genetic parameter estimates for wood stiffness, strengh, internal checking and resin bleeding for radiata pine. Can. J. For. Res. 34:2601-2610.

Maclaren, P. 2002. Wood quality of the pine on form sites a review of the issues. Forest \& Farm Plantation Management Cooperative. Piers Maclaren \& Associates Ltd, Report No 80, 3pp.

McConchie, D.L.1999. An update on within-ring internal checking including a procedure to identify logs prone to form check during drying. Proceedings of $3^{\text {rd }}$ Wood Quality Symposium. "Emerging Technologies for Evaluating Wood Quality for Wood Processing". New Zealand Forest Research Institute, Rotorua. 
Miller, W.; Simpson I. 1992. Collapse associated internal checking in radiata pine. $3^{\text {rd }}$ IUFRO International Wood Drying Conference, 298-398.

Montgomery, D. 1996. Diseño y análisis de experimento. Capitulo 7. Grupo Editorial Iberoamericana, México.

Olykan, S. 2004. Forest nutrition- boron. Southern Tablelands Farm Forestry Network Newsletter, $2 \mathrm{pp}$.

Pang, S.; Orchard, R.; McConchie, D. 1999. Tangential shrinkage of Pinus radiata earlywood and latewood, and its implication for within-ring internal checking. New Zealand Journal of Forestry Science 29(3):484-491.

Potaczki, T.L.; Nair, H.; Butterfield, B.; Jackson, S.L. 2006. Intra-ring checking in Pinus radiate D. Don: the occurrence of cell wall fracture, cell collapse and lignin distribution. Trees 21:221-229.

Simpson, I.; Booker, R.E.; Haslett, A.N. 2002. Within-ring internal checking. Wood Proces News 32: $1-3$

Vera, J. 2005. Mejoramiento del secado industrial del pino radiata para remanufactura. Estudio de grietas internas. Proyecto de Título, Ingeniería Civil en Industrias Forestales, Departamento de Ingeniería en Maderas, Facultad de Ingeniería, Universidad del Bío-Bío. Concepción, Chile. 50 p. 
